PROCEEDINGS OF THE

AMERICAN MATHEMATICAL SOCIETY

S 0002-9939(03)06996-X

Article electronically published on May 9, 2003

\title{
THE TORIC COBORDISMS
}

\author{
ALEXANDRA MOZGOVA
}

(Communicated by Ronald A. Fintushel)

\begin{abstract}
We introduce the notions of oriented and unoriented cobordisms in the class of closed 3-manifolds fibered by tori $T^{2}$ and compute the corresponding cobordism groups.
\end{abstract}

Two smooth compact $n$-manifolds, $M$ and $N$, are said to be cobordant if their disjoint union $M \sqcup N$ is diffeomorphic to the boundary of a smooth compact $(n+1)$ manifold $W$. Cobordism is an equivalence relation on the set of smooth compact $n$-manifolds, and the set of equivalence classes admits the structure of an abelian group in which the operation is induced by disjoint union. Other cobordism groups are obtained by restricting the cobordism relation to classes of manifolds with additional structure, such as an orientation, or a complex structure or a spin structure. In this note we will consider the cobordism groups of smooth compact $n$-manifolds which admit fibrations for which the fiber is a 2-dimensional torus.

An $n$-dimensional torus bundle is a smooth fibration $f: M^{n} \rightarrow B^{n-2}$ where the total space $M$ and the base $B$ are smooth compact manifolds of dimension $n \geq 2$ and $n-2$ respectively, and where the fibers of $f$ are diffeomorphic to the 2-dimensional torus $T^{2}$. By an oriented torus bundle we mean a torus bundle $f: M \rightarrow B$ together with a choice of an orientation of $M$. If an (oriented) torus bundle $f: M \rightarrow B$ is denoted by $M$, then $\partial M$ will denote the (oriented) torus bundle obtained by restricting $f$ to the boundary of $M$ (with the orientation induced from that of $M)$; we will use $M^{-}$to denote the oriented torus bundle consisting of the fibration $f: M \rightarrow B$ together with the opposite choice of orientation of $M$. If $M_{1}$ and $M_{2}$ are (oriented) torus bundles, then we will write $M_{1}=M_{2}$ if there is a fiber-preserving (orientation-preserving) diffeomorphism from $M_{1}$ to $M_{2}$.

Two $n$-dimensional torus bundles $M_{1}$ and $M_{2}$ are said to be toric cobordant if there exists an $(n+1)$-dimensional torus bundle $W$ such that $\partial W=M_{1} \sqcup M_{2}$. If $M_{1}$ and $M_{2}$ are oriented torus bundles, then we will say that they are oriented toric cobordant if there exists an oriented torus bundle $W$ such that $\partial W=M_{1} \sqcup M_{2}^{-}$. In either case $W$ will be called a cobordism from $M_{1}$ to $M_{2}$. Toric cobordism and oriented toric cobordism are equivalence relations. We will use the notation $M_{1} \sim M_{2}$ to indicate that the (oriented) torus bundles $M_{1}$ and $M_{2}$ are (oriented) toric cobordant, and in either case the equivalence class of $M$ will be denoted $M^{\omega}$. The equivalence classes of $n$-dimensional torus bundles under toric cobordism form

Received by the editors March 1, 2001 and, in revised form, August 23, 2002.

2000 Mathematics Subject Classification. Primary 57M50, 57M07; Secondary 55R10.

Key words and phrases. Torus bundles over circle, toric cobordism.

This work was supported by French Government Grant \#19981314. 
an abelian group $\Omega_{n}^{T^{2}}$,unor under the operation defined by $M_{1}^{\omega}+M_{2}^{\omega}=\left(M_{1} \sqcup M_{2}\right)^{\omega}$. The equivalence classes of $n$-dimensional oriented torus bundles under oriented toric cobordism form an abelian group $\Omega_{n}^{T^{2}}$,or under the operation defined by $M_{1}^{\omega}+$ $M_{2}^{\omega}=\left(M_{1} \sqcup M_{2}^{-}\right)^{\omega}$. The unoriented toric cobordism groups of $n$-manifolds are isomorphic to the unoriented bordism groups $\Omega_{n-2}(B G L(2, \mathbb{Z}))$ of maps from $B^{n-2}$ to $B \operatorname{Diff}\left(T^{2}\right)$. Lemma 1 below shows that the oriented toric cobordism groups of 3 -manifolds are isomorphic to the oriented bordism groups $\Omega_{1}(B G L(2, \mathbb{Z})$ ) (pointed out by A. Marin).

The rest of this note will be devoted to the proof of the following:

Theorem 1. The third group of oriented toric cobordisms $\Omega_{3}^{T^{2} \text {,or }}$ is isomorphic to $\mathbb{Z}_{12}$ and is generated by the class of the torus bundle with the monodromy $\left(\begin{array}{ll}1 & 1 \\ 0 & 1\end{array}\right)$.

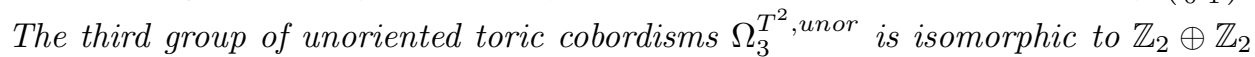
and is generated by the classes of the torus bundles with the monodromies $\left(\begin{array}{cc}0 & -1 \\ 1 & 0\end{array}\right)$ and $\left(\begin{array}{ll}0 & 1 \\ 1 & 0\end{array}\right)$.

\section{THE TORIC COBORDISMS OF 3 -MANIFOLDS}

The quotient $M_{\varphi}=T^{2} \times I /(x, 0) \sim(\varphi(x), 1)$ where $\varphi$ is a diffeomorphism of $T^{2}$ is a torus bundle over the circle with monodromy $\varphi$. If the diffeomorphisms $\varphi_{1}, \varphi_{2}$ are isotopic, then $M_{\varphi_{1}}=M_{\varphi_{2}}$. A diffeomorphism of $T^{2}$ is determined up to isotopy by its induced map on the first integral homology group, and hence the diffeotopy group is isomorphic to $G L(2, \mathbb{Z})$.

Let $G$ denote the group $G L(2, \mathbb{Z})$; let $G^{\prime}$ be its commutator subgroup and $G^{2}$ be the subgroup of $G$ generated by the squares of the elements of $G$.

Claim 1. Let $M_{\varphi}$ be a 3-dimensional torus bundle defined as above. There exists a 4-dimensional manifold $W$ fibered by tori over an orientable surface with $\partial W=M_{\varphi}$ if and only if $\varphi \in G^{\prime}$. Such a $W$ is orientable if and only if $\varphi$ can be written as $\varphi=\prod_{j=1}^{g}\left[\varphi_{2 j-1}, \varphi_{2 j}\right]$ where $\operatorname{det} \varphi_{i}=1$ for $i=1, \ldots, 2 g$.

Proof. The $T^{2}$-bundles over a finite cell complex $X$ are classified by homotopy class of maps from $X$ to BDiff $\left(T^{2}\right)$. Such a class determines a conjugacy class of homomorphisms

$$
\pi_{1}(X) \rightarrow \pi_{1}\left(B \operatorname{Diff}\left(T^{2}\right)\right) \cong \pi_{0}\left(\operatorname{Diff}\left(T^{2}\right)\right) \cong G .
$$

In our case $X$ is a surface with non-empty boundary, so $\pi_{1}(X)$ has cohomological dimension 1 (being free). Thus $T^{2}$-bundles over a surface with non-empty boundary are in bijection with the homomorphisms $\pi_{1}(X) \rightarrow G$.

The manifold $W$ we are looking for exists if and only if there is a commutative diagram

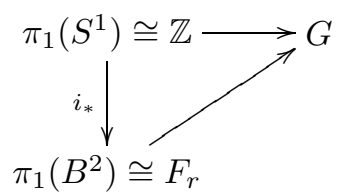

where $i_{*}$ is induced by inclusion of the boundary, i.e. $\operatorname{Im} i_{*} \subset\left(F_{r}\right)^{\prime}$, hence we have shown the first part of the claim.

To see when $W$ is orientable, use the standard construction of an oriented surface from the disk $D^{2}$, by identifying some 1-disks $I_{i}$ on its boundary. Then we can 
explicitly construct $W$ starting from $D^{2} \times T^{2}$ by gluing $\left(I_{i} \times T^{2}\right)$ 's on its boundary. As $D^{2} \times T^{2}$ is oriented and the gluing must create no orientation-reversing loop, it is not hard to see that the condition $\operatorname{det} \varphi_{i}=1$ assures the orientability of $W$.

Reasoning similarly, we get for fiber bundles over a non-orientable surface

Claim 2. Let $M_{\varphi}$ be a 3-dimensional torus bundle. There exists a 4-dimensional manifold $W$ fibered by tori over a non-orientable surface with $\partial W=M_{\varphi}$ if and only if $\varphi \in G^{2}$. Such a $W$ is orientable if and only if $\varphi$ can be written as $\varphi=\prod_{j=1}^{k} \varphi_{j}^{2}$ where $\operatorname{det} \varphi_{i}=-1$ for $j=1, \ldots, k$.

Claim 3. Let $\varphi_{1} \ldots \varphi_{n} \in G=G L(2, \mathbb{Z})$ and $M_{\varphi_{1}}, \ldots, M_{\varphi_{n}}$ be the corresponding 3dimensional torus bundles. There exists a 4 -dimensional manifold $W$ fibered by tori over an orientable surface with $\partial W=M_{\varphi_{1}} \sqcup \ldots \sqcup M_{\varphi_{n}}$ if and only if $\prod_{i=1}^{n} \varphi_{i} \in G^{\prime}$.

Proof. The proof is immediately obtained from Claim 1 and the fact that the disjoint union $M_{\varphi_{1}} \sqcup \ldots \sqcup M_{\varphi_{n}}$ is cobordant to $M_{\psi}$ with $\psi=\prod_{i=1}^{n} \varphi_{i}$, by a toric cobordism with base the sphere $S^{2}$ with $n+1$ holes (it can be constructed similarly to the proof of Claim 1).

Claim 4. For $\varphi_{1} \ldots \varphi_{n} \in G$ and $M_{\varphi_{1}}, \ldots, M_{\varphi_{k}}$ as above, there exists a 4-dimensional manifold $W$ fibered by tori over a non-orientable surface with boundary $\partial W=$ $M_{\varphi_{1}} \sqcup \ldots \sqcup M_{\varphi_{n}}$ if and only if $\prod_{i=1}^{n} \varphi_{i} \in G^{2}$.

Lemma 1. There exists an oriented toric cobordism with an orientable base between $M_{\varphi}$ and $M_{\psi}$ if and only if there also exists an oriented toric cobordism with a nonorientable base between them.

Proof. By $G_{-}^{2}$ we denote the subgroup of $G L(2, \mathbb{Z})$ generated by the squares of matrices with negative determinant

$$
G_{-}^{2}=\left\langle\left\{a_{1}^{2} a_{2}^{2} \ldots a_{k}^{2} \mid \operatorname{det} a_{i}=-1\right\}\right\rangle .
$$

It is evident that $G_{-}^{2} \subset S L(2, \mathbb{Z})$ and is normal in it. We show that $G_{-}^{2}=$ $(S L(2, \mathbb{Z}))^{\prime}$ and this implies the claim. We use the following presentations of $G L(2, \mathbb{Z})$ and $S L(2, \mathbb{Z})$; see $(\underline{5}, 2.23)$. For $A=\left(\begin{array}{cc}0 & -1 \\ 1 & 0\end{array}\right), B=\left(\begin{array}{cc}0 & 1 \\ -1 & 1\end{array}\right)$ and $R=$ $\left(\begin{array}{ll}0 & 1 \\ 1 & 0\end{array}\right)$

$$
\begin{gathered}
G L(2, \mathbb{Z})=\left\langle A, B, R \mid A^{2}=B^{3}, A^{4}=R^{2}=(R A)^{2}=(R B)^{2}=1\right\rangle, \\
S L(2, \mathbb{Z})=\left\langle A, B \mid A^{2}=B^{3}, A^{4}=1\right\rangle .
\end{gathered}
$$

The commutator subgroup $(S L(2, \mathbb{Z}))^{\prime}$ of $S L(2, \mathbb{Z})$ is a free group of rank 2 generated by $[A, B]=\left(A R B^{-1}\right)^{2} \in G_{-}^{2}$ and $\left[A, B^{-1}\right]=\left(B^{-1} R B\right)^{2} \in G_{-}^{2}$. Thus $(S L(2, \mathbb{Z}))^{\prime} \subset G_{-}^{2}$, and as $(S L(2, \mathbb{Z}))^{\prime} \triangleleft S L(2, \mathbb{Z})$, we have $(S L(2, \mathbb{Z}))^{\prime} \triangleleft G_{-}^{2}$.

By using the relations $R A=A^{-1} R$ and $R B=B^{-1} R$, each element $a \in G L(2, \mathbb{Z})$ can be written in the normal form $R^{\varepsilon} A^{k_{1}} B^{l_{1}} \ldots A^{k_{n}} B^{l_{n}}$ where $\varepsilon \in\{0 ; 1\}$. If det $a=$ -1 , the element $a$ can be written in the form $R A^{k_{1}} B^{l_{1}} \ldots A^{k_{n}} B^{l_{n}}$.

Now $G_{-}^{2} /(S L(2, \mathbb{Z}))^{\prime}=\left\langle\left\{\left(R A^{i} B^{j}\right)^{2} \mid A^{2}=B^{3},(R A)^{2}=(R B)^{2}=A^{4}=R^{2}=\right.\right.$ $1, A B=B A\}\rangle=1$. Thus, $G_{-}^{2}=(S L(2, \mathbb{Z}))^{\prime}$.

Proof of Theorem 1. It follows from Lemma 1 that for $\varphi, \psi \in S L(2, \mathbb{Z})$ there exists an oriented toric cobordism between the torus bundles $M_{\varphi}$ and $M_{\psi}$ if and only if 


$$
\begin{aligned}
& \varphi \psi^{-1} \in(S L(2, \mathbb{Z}))^{\prime} \text {. Thus } \\
& \quad \Omega_{3}^{T^{2}, \text { or }} \cong S L(2, \mathbb{Z}) /(S L(2, \mathbb{Z}))^{\prime}=\left\langle A, B \mid A^{2}=B^{3}, A^{4}=1, A B=B A\right\rangle \cong \mathbb{Z}_{12} .
\end{aligned}
$$

The generator of $\Omega_{3}^{T^{2}, \text { or }}$ is the conjugacy class of the element $B^{-1} A^{-1}=\left(\begin{array}{ll}1 & 1 \\ 0 & 1\end{array}\right)$.

The subgroup $G^{\prime}$ lies in the subgroup $G^{2}$. Thus, if there exists an unoriented toric cobordism with an orientable base between $M_{\varphi}$ and $M_{\psi}$, then there also exists an unoriented toric cobordism with a non-orientable base between them. Hence, Claim 4 lets us calculate the third group of unoriented toric cobordims, $\Omega_{3}^{T^{2}}$,unor , as well. Thus,

$$
\begin{gathered}
\Omega_{3}^{T^{2}, \text { unor }} \cong G / G^{2}=\langle A, B, R| A^{2}=B^{3}, A^{4}=R^{2}=(R A)^{2}=(R B)^{2}=1, \\
\left.A B=B A, A R=R A, B R=R B, A^{2}=B^{2}=1\right\rangle \cong \mathbb{Z}_{2} \oplus \mathbb{Z}_{2} .
\end{gathered}
$$

The generators here are the conjugacy classes of $A=\left(\begin{array}{cc}0 & -1 \\ 1 & 0\end{array}\right)$ and $R=\left(\begin{array}{ll}0 & 1 \\ 1 & 0\end{array}\right)$.

Remark 1. The toric cobordism is a cobordism of manifolds with some fixed torus bundle structures. In some cases, for $M_{\varphi}, M_{\psi}$ that are not oriented toric cobordant, we can choose other torus bundle structures on their total spaces in such a way that they become oriented toric cobordant. For example, take a $\varphi \in S L(2, \mathbb{Z})$ such that $\varphi^{2} \nsubseteq(S L(2, \mathbb{Z}))^{\prime}$. Then, the corresponding $M_{\varphi}$ is not oriented toric cobordant to $M_{\varphi}^{-}$, but is oriented toric cobordant to $M_{\gamma \varphi \gamma^{-1}}$ for $\gamma$ with $\operatorname{det} \gamma=-1$. The reason for this is that the total spaces of $M_{\varphi}$ and $M_{\gamma \varphi \gamma^{-1}}$ are homeomorphic by a fiber preserving homeomorphism inducing the orientation reversing map of the basis $S^{1}$ (see [1] or [3]).

Remark 2. Let $\varphi \in S L(2, \mathbb{Z})$ and $W^{4}$ be a toric cobordism between $M_{\varphi}$ and $\varnothing$ that has an orientable base of genus $g$. Then $\varphi$ is a product of $g$ commutators. By taking $\psi^{\prime}, \psi^{\prime \prime}$ such that $\left[\psi^{\prime}, \psi^{\prime \prime}\right]=1$, one can write $\varphi$ as a product of $g+1$ commutators and so one can construct another toric cobordism between $M_{\varphi}$ and $\varnothing$ with orientable base of genus $g+1$. Thus, quite naturally we come to the following question: what is the minimal genus of the orientable base of $W^{4}$ ?

For this, we can utilize Culler's algorithm ([2]) which determines, for finite groups $A$ and $B$ and $a \in(A * B)^{\prime}$, the minimal number of elements of $A * B$ required to represent $a$ as a product of their commutators. In order to extend Culler's algorithm from free products to $S L(2, \mathbb{Z})=\mathbb{Z}_{4} *_{\mathbb{Z}_{2}} \mathbb{Z}_{6}$, consider the projection homomorphism $\alpha: \mathbb{Z}_{4} * \mathbb{Z}_{2} \mathbb{Z}_{6} \rightarrow \mathbb{Z}_{2} * \mathbb{Z}_{3}$. As Culler's algorithm can be applied to the group $\mathbb{Z}_{2} * \mathbb{Z}_{3}$, it remains to note that the restriction of the homomorphism $\alpha$ to the commutator subgroup

is an isomorphism.

$$
\alpha^{\prime}=\left.\alpha\right|_{\left(\mathbb{Z}_{4} * \mathbb{Z}_{2} \mathbb{Z}_{6}\right)^{\prime}}:\left(\mathbb{Z}_{4} * \mathbb{Z}_{2} \mathbb{Z}_{6}\right)^{\prime} \longrightarrow\left(\mathbb{Z}_{2} * \mathbb{Z}_{3}\right)^{\prime}
$$

\section{ACKNOWLEDGMENT}

The author is grateful to Gilbert Levitt for pointing out Culler's paper. It is also a pleasure to thank the referee for constructive comments.

\section{REFERENCES}

1. G. Burde, H. Zieschang, A topological classification of certain 3-manifolds, Bulletin of AMS, 74 (1968), pp.122-124 MR 36:2153

2. M. Culler, Using surfaces to solve equations in free groups, Topology 20 (1981), pp.133-145 MR 82c:20052 
3. A. Hatcher, Notes on 3-manifold Topology, available on http://math.cornell.edu/ hatcher

4. H. Zieschang, On toric fiberings over surfaces, Math. Notes 5 (1969), pp.341-345

5. H. Zieschang, Finite groups of mapping classes of surfaces, Springer-Verlag, Berlin Heidelberg New York, 1980, 1981 MR 86g:57001

Laboratoire Emile Picard CNRS UMR 5580, Université Paul Sabatier Toulouse III, 118, route de Narbonne, 31077 Toulouse, France - And - Institute of Mathematics of Ukrainian National Academy of Science, vul. Tereschenkivska, 3, 252601 Kiev, Ukraine E-mail address: mozgova@picard.ups-tlse.fr 